\title{
EMPRESARIALIDAD DE NUEVAS EMPRESAS DE HUACHO Y HUAMANGA
}

MARÍA CELINA HUAMÁN MEJÍA*

E-mail: cmarihmehotmail.com

\begin{abstract}
RES UMEN
Objetivos: Identificar los principales factores que influyen en la creación y avance de empresas dentro de diferentes contextos locales (Huamanga y Huacho), así como conocer y evaluar los factores que afectan el éxito de las nuevas empresas que allí surgen, para identificar las acciones que los gobiernos pueden llevar a cabo. Método: Se aplicaron cuestionarios a 81 fundadores de Huacho y Huamanga con empresas en los sectores, basados en el conocimiento y manufactura. Resultados: En Huacho los emprendedores son predominantemente mujeres y en Huamanga son varones, con una edad promedio de 31 años, con estudios superiores no concluidos y concluidos. Fundaron sus empresas cuando tenían de 20 a 30 años, usando principalmente ahorros personales y préstamos bancarios. De su experiencia laboral los emprendedores obtienen motivación, ideas, competencias y habilidades empresariales, y lo más importante: contactos, que le sirven de base para la puesta en marcha de sus negocios. El entorno de negocios de Huamanga es más difícil que el de Huacho debido a la escasez de financiamiento, la complejidad burocrática y los altos costos impositivos y regulatorios. Las nuevas empresas de Huacho crecen más rápido y logran mayores escalas que las de Huamanga y también cuentan con redes de contactos más estables. La proporción entre nuevas empresas basadas en el conocimiento y empresas exportadoras es más alta en Huacho que en Huamanga. Conclusión: Los principales factores que motivan el ingreso de los empresarios en el mundo de negocios son el mejoramiento de sus ingresos, el deseo de autorealización y el anhelo de ser su propio jefe. Los factores que limitan el avance son las dificultades de financiamiento con mayor incidencia en Huamanga, y en ambas localidades predomina la falta de asesoramiento para resolver problemas, la búsqueda de nuevos clientes y la contratación de trabajadores calificados.
\end{abstract}

Palabras clave: Emprendedores, empresas, desarrollo, Huacho y Huamanga.

\section{ABSTRACT}

Objetives: This study has as an objective to identify the principal factors that influence on the process of formation of new enterprises and the advancement within different local contexts, as well as how to know and evaluate the factors that affect the success of new enterprises in

\footnotetext{
* Magíster en Administración. Profesora Auxiliar de la Facultad de Ciencias Administrativas de la UNMSM.
} 
Huacho and Huamanga in order to priorize the actions that the governments have to do to promote the enterprising. Method: So as to know the factors that affect the success in the new enterprises and to learn how the entrepreneurs can manage their businesses, 81 manufacturing owners were interviewed in Huacho and Huamanga. Results: There are important contrasts: The entrepreneurs are specially 31 year-old women in Huacho and men in Huamanga, whose high-level studies were concluded or not. These people formed their enterprises when they were between 20 and 30 years of age, using personal savings to open their business. They got experience, competence, business abilities and above all long lasting contacts. The business out of Huamanga is more difficult than the business in Huacho due to the lack of finance, the bureocracy and the high costs. Conclusion: It is inferred that the areas that require policies to promote enterprising and the peculiarities of the enterpreneuring process in each region consider the following aspects: one is the global aspect over the objectives and the strategic importance of the supporting policies to the enterprising and the other is on how to promote enterprises in the studied areas of Huacho and Huamanga.

Keywords: Entrepreneurs, enterprises, development, Huacho and Huamanga.

\section{INTRODUCCIÓN}

Las nuevas empresas contribuyen de manera significativa al crecimiento económico, lo cual es especialmente beneficioso para los países en desarrollo. Los emprendedores que 10gran crear nuevas empresas generan empleo, expanden segmentos del mercado, incrementan la producción de bienes y servicios, y dan mayor dinamismo a las áreas donde operan. A diferencia de la tendencia en otras regiones del mundo, el desarrollo empresarial de América Latina es elitista, individualista, de escasa innovación y poca visión globalizadora, según revela un informe elaborado conjuntamente por el Banco Interamericano de Desarrollo (BID) y FUNDES Internacional. El menor dinamismo de las nuevas empresas latinoamericanas, en comparación con firmas creadas en el sureste asiático - la Europa mediterránea, está estrechamente relacionado con restricciones culturales de los sistemas de emprendimiento en nuestra región, marcadas diferencias culturales y motivacionales, así como los modelos de organización productiva imperantes.

Un factor que enciende luces rojas en el desarrollo económico latinoamericano, que tiene una población predominantemente femenina, es que las mujeres son menos emprendedoras que los hombres y con mayor temor al riesgo ${ }^{1}$. El estudio presenta nuevos elementos sobre la capacidad de los empresarios para crear puestos de trabajo y contribuir al dinamismo de los negocios y los mercados internos. Asimismo demuestra también contrastes interesantes entre América Latina y el Este de Asia, donde las nuevas empresas crecen con mayor rapidez y alcanzan mayores escalas productivas que en nuestra región. Así mismo las nuevas empresas hacen uso más intensivo de las tecnologías del conocimiento y tienen mayor inserción en los mercados internacionales que las de América Latina ${ }^{2}$.

El presente estudio tiene como objetivo identificar los principales factores que influyen en el proceso de creación y avance de empresas dentro del contexto de las ciudades de Huacho y Huamanga, para priorizar y destacar las acciones que el gobierno y las organizaciones podrían llevar acabo para promover la empresarialidad. ¿Cuáles son los factores más influyentes que incentivan o delimitan la entrada y el avance de los empresarios en dicho proceso, dentro de ambos contextos? La investigación sirve de referencia básica para delinear políticas basadas en la creación y desarrollo de nuevas empresas, y esperamos que los hallazgos de este trabajo inspiren a los programas dirigidos a la promoción de la empresarialidad ${ }^{3}$.

\section{MARCO TEÓRICO}

El estudio de la empresarialidad encierra la necesidad de introducir profundos cambios en el enfoque convencional. A pesar que el tra- 
bajo pionero de Schumpeter (1965) determinó que la riqueza es creada por los emprendedores y reconoce el papel de la empresarialidad como motor de la innovación y el desarrollo económi$\mathrm{co}$, los economistas han tendido, tradicionalmente, a concentrarse en el análisis de la función económica del empresario más que en la explicación del proceso de emergencia de nuevas empresas ${ }^{4}$.

Una corriente pionera de trabajos se ha concentrado en estudiar «quién es un empresario» o cuáles son sus atributos de personalidad. Mc Clelland (1961) los caracteriza, fundamentalmente, por su necesidad de logro:

"Los emprendedores son individuos con visión empresarial, que manifiestan una conducta y orientan su comportamiento al desarrollo y surgimiento del espíritu emprendedor interno; generando y aprovechando ideas innovativas; desarrollándolas como oportunidades de negocio rentable; comprometiendo su tiempo y esfuerzo en investigar, crear y moldear esas ideas para convertirlas en negocios para su propio beneficio y creatividad de bienes y servicios, convirtiéndolas así en un agente de cambio» ${ }^{5}$.

Gilder (1984), por su parte, describe a los empresarios como personas capaces de aprender, que persiguen sus objetivos a pesar de sus fracasos y las frustraciones, y que, finalmente, obtienen el éxito al romper viejos patrones y crear su propio nuevo orden. Estos individuos eligen asumir el riesgo y comenzar su propia empresa debido a que consideran que el mercado responderá y valorizará su idea mejor que la propia organización en la que trabajan ${ }^{6}$.

Helcome (1998) presenta una teoría de la relación económica entre empresarialidad y crecimiento económico, en la cual el empresario es el motor interno del crecimiento ${ }^{7}$. Schumpeter (1965) describe la actividad emprendedora como la destrucción creativa, y pone a los emprendedores en el centro de su teoría. Las empresas existentes, si aspiran a permanecer, deben entrar al juego de destrucción creativa y desarrollar constantemente nuevos paradigmas y modelos de negocios ${ }^{8}$. Bygrave (1999) hace una analogía entre los dinosaurios y las organizaciones incapaces de evolucionar: ambas se extinguen al fallar en su intento por adaptarse ${ }^{9}$. Timmons y Spinell (2003) proponen un simple e interesante modelo que se basa en tres fuer- zas: las oportunidades, los recursos y el equipo, las cuales deben articularse adecuadamente y estar en perfecto balance ${ }^{10}$.

Se ha desarrollado un método «ecléctico» para la comprensión del fenómeno, identificando fases en el proceso de creación de empresas (gestación del proyecto, puesta en marcha y desarrollo inicial), y dentro de estas fases conceptuales se incluyen elementos que inciden y determinan las condiciones de empresarialidad, que son cultura y sistema educativo, estructura y dinámica productiva, condiciones económicas y de los mercados, redes, marco regulatorio y aspectos personales. Evidentemente no todas estas circunstancias objetivas que afectan la creación de empresas ni todas las etapas del proceso pueden ser cubiertas, mejoradas o desarrolladas por actividades de formación, aunque muchas de ellas pueden abordarse a partir de la puesta en marcha de programas de capacitación ${ }^{11}$.

\section{MÉTODOS Y RESULTADOS}

\subsection{Métodos}

El enfoque de la investigación es cuantitativo (por encuestas); el universo y ámbito: las provincias de Huamanga y Huacho; el contexto de investigación: ficha técnica de las empresas estudiadas.

- Tamaño de la muestra: 81 fundadores de empresas, 46 en Huacho y 35 en Huamanga.

- Fecha de encuesta: entrevistas personales realizadas entre setiembre y noviembre del 2004.

- Muestras en cada ciudad: más de 10 nuevas empresas dinámicas y al menos 3 menos dinámicas (grupo control) en cada ciudad estudiada.

- Ciudades estudiadas: Huamanga y Huacho.

- Áreas locales: Vegeta, Sayán, Huacho y Huaura (Huacho), Jesús Nazareno, San Juan Bautista, Carmen Alto y Ayacucho (Huamanga).

- Sectores: tradicional manufacturero: 47\% en Huacho y 68\% en Huamanga; basado en el conocimiento: 53\% en Huacho y 37\% en Huamanga.

- Formalización: todas las empresas encuestadas están registradas ante las autoridades correspondientes, no se incluyeron microempresas informales.

- Destino de las ventas: solo el mercado interno: 62\% en Huacho y $82 \%$ en Huamanga; exportaciones: $38 \%$ en Huacho y $16 \%$ en Huamanga. 
- Validación de cuestionarios: más del 90\% de las preguntas debían estar contestadas.

- Instrumentos: Se utilizó un cuestionario estructurado y estandarizado que contiene preguntas cerradas y de escala. Se supervisó el 45\% de la cuota de cada entrevistador utilizado. Para el análisis de los datos se utilizaron porcentajes y promedios de las características.

\subsection{Resultados}

El resultado analiza tres etapas críticas del proceso de creación de empresas: gestación, puesta en marcha y desarrollo inicial. Para cada una de esas etapas se describen las motivaciones, el proceso de toma de decisiones, la búsqueda de oportunidades, la movilización de recursos y los principales problemas.

El periodo de gestación es más largo ya que va desde el momento en que el empresario tiene la motivación, identifica la oportunidad y concibe la empresa hasta cuando comienza a hacer planes concretos para poner la idea en marcha. La fase de puesta en marcha transcurre desde que la persona decide establecer una empresa hasta cuando reúne los medios necesarios para inaugurarla. La tercera etapa, de lanzamiento o desarrollo inicial, es la que cubre el periodo de los tres primeros años de funcionamiento, los cuales son críticos para la supervivencia de la empresa.

En ambas localidades las nuevas empresas contribuyen al crecimiento económico, aunque su expansión es más robusta en Huacho. Esto puede observarse en la evolución de la facturación de las nuevas empresas: las empresas de Huacho tienen mayores ventas desde el primer año de existencia y crecen mucho más rápido que las de Huamanga. Aunque los ingresos totales de las nuevas empresas son bajos al comienzo en ambas localidades, el volumen de facturación de los negocios del norte chico es superior al de los huamanguinos. Con el transcurso del tiempo estas diferencias se mantienen: después de tres años de operación las empresas de Huacho venden más que las empresas de Huamanga, superando el 19\% de ellas los 20000 dólares anuales, mientras que el 14\% de las empresas en Huamanga alcanzan ese valor, en el mismo periodo de tiempo. La expansión de la fuerza de trabajo en el primer año en Huamanga es del 14\%, mientras que en Huacho es del 83\%.
- La etapa de gestación. Los fundadores encuestados informaron que desde el momento en que sienten el deseo de iniciar una empresa hasta cuando identifican la oportunidad de negocios, pasan un promedio de uno a dos años, tanto para el caso de Huamanga como para el de Huacho.

Las motivaciones que dan origen a las nuevas empresas incluyen objetivos netamente económicos como el desarrollo personal. En Huacho como en Huamanga «la mejora de ingresos» es el principal motivo para crear un negocio, y se cita con mayor frecuencia la autorrealización como un objetivo de tipo no económico. El segundo factor no económico más importante es enfrentar desafíos continuos. El apoyo familiar también es un aspecto positivo en la motivación de los empresarios: la gran mayoría de las personas que fundaron nuevas empresas recibieron apoyo de su núcleo familiar y grupo social. Solamente el 21\% de los empresarios huamanguinos y alrededor de 27\% de los huachanos encontraron reacciones negativas de sus familiares cuando dieron a conocer su interés en crear una empresa (Gráfico 1).

La experiencia laboral es la fuente de movilización y creación de competencias empresariales más importantes para los empresarios de ambas regiones. Los modelos de empresarios ejemplares (modelos de rol) ejercen un efecto motivacional destacado para los fundadores de empresas en Huacho, mientras que en Huamanga los medios de comunicación masivos ejercen un rol importante en su difusión. La formación universitaria es cierto que provee de conocimientos técnicos a los emprendedores, pero tiene una limitada influencia en cuanto a la generación de motivación y competencias empresariales.

La principal fuente de oportunidades de negocios está ligada a la venta de servicios a empresas de pequeña escala. En la mayoría de los casos se trata de servicios basados en el conocimiento, resaltando servicios relacionados con internet, que representa un mercado con demanda creciente. La gran mayoría de las nuevas empresas tanto en manufactura como en servicios basados en el conocimiento atiende mercados internos; en ambas regiones son numerosas las empresas relacionadas con servicios de Internet. En cuanto a exportaciones, después de unos cinco años, alrededor del 7\% de las empresas huachanas logra hacerlo. 


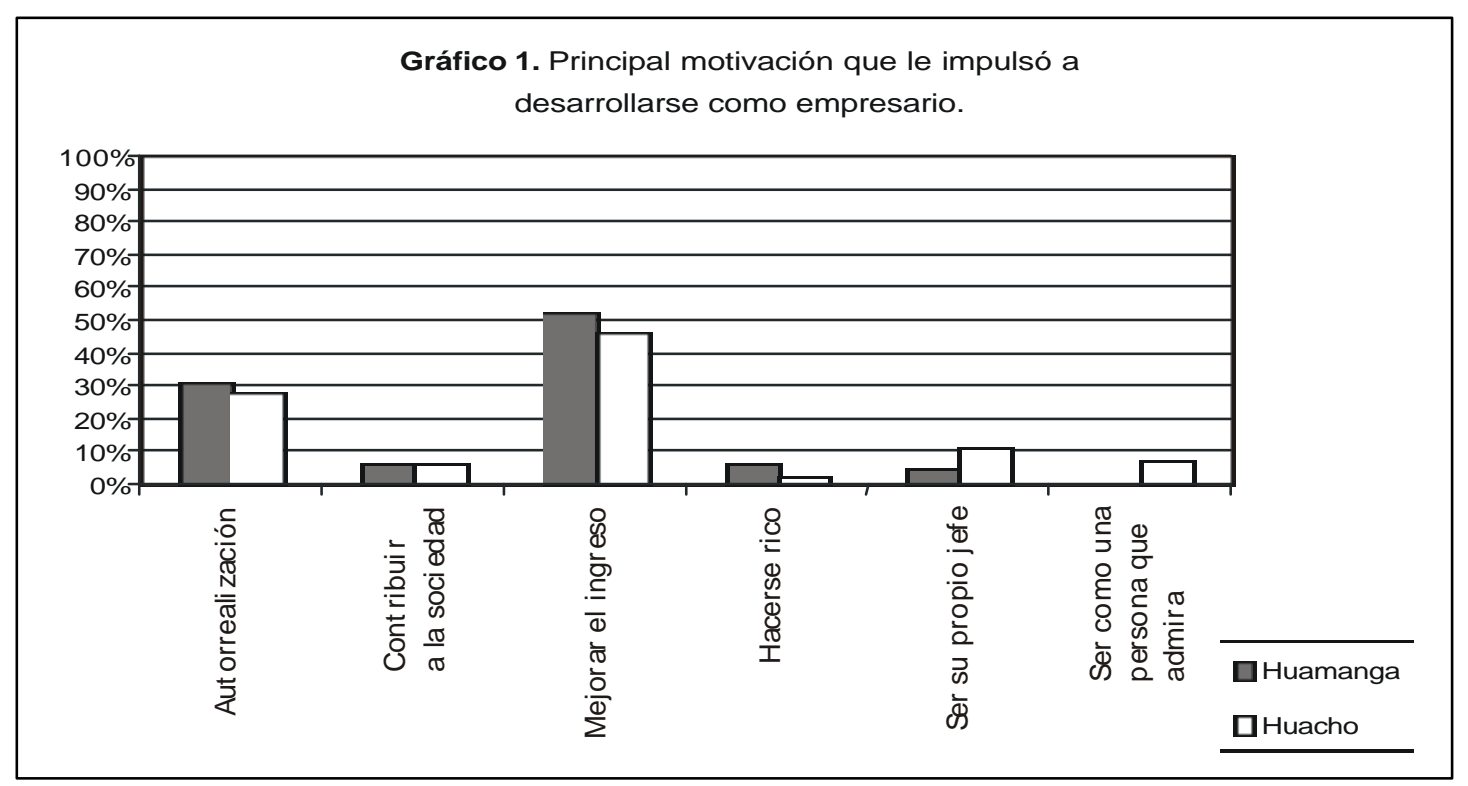

Las redes y contactos con el entorno social del emprendedor y sus socios, son otro de los factores más importantes en el desarrollo de las nuevas empresas. Más del 51\% de los empresarios en Huamanga señalan que la «experiencia laboral previa» y la discusión con otros jugaron un papel importante para identificar la oportunidad de negocio. En Huacho resaltan los contactos del entorno social y contactos personales específicos (Gráfico 2).
- La etapa de puesta en marcha. La decisión del fundador de poner en marcha una nueva empresa está influenciada fuertemente por motivaciones que reflejan objetivos económicos y no económicos, similares a los de la etapa de gestación. Las motivaciones personales más importantes son «la autorrealización personal», «ser su propio jefe»y «mejorar el ingreso». Estas motivaciones prevalecen en ambas localidades (Gráfico 3).

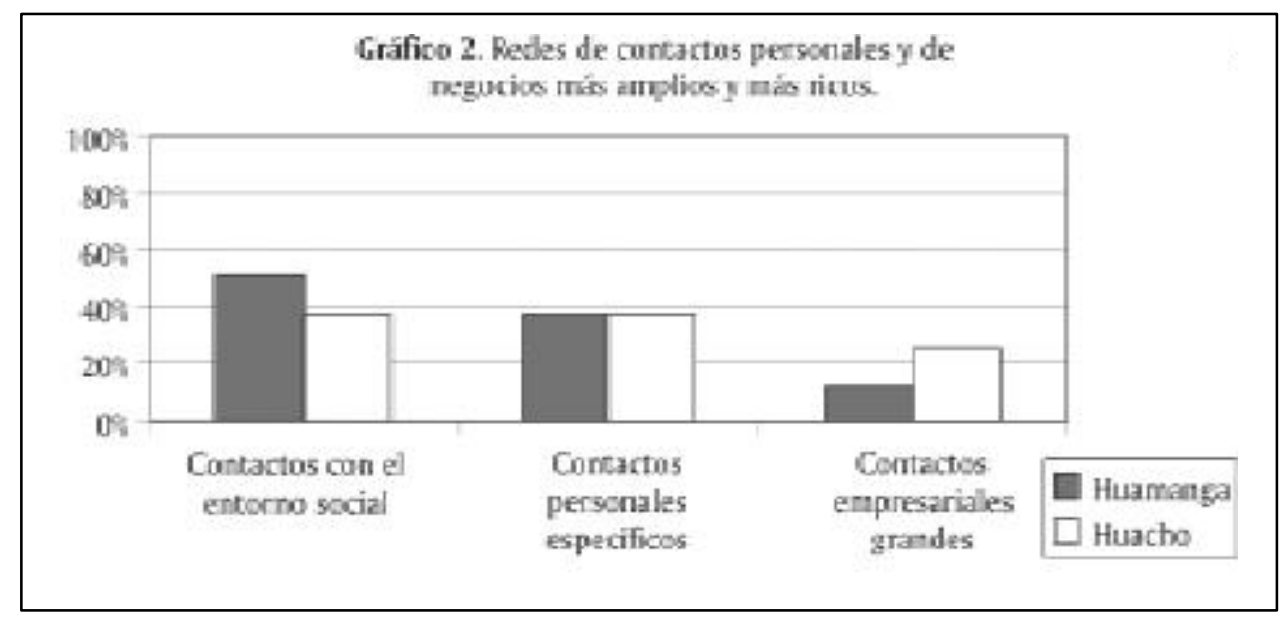

Los empresarios, incluyendo los líderes de las empresas más dinámicas, no desarrollan mayoritariamente actividades de planificación estratégica. Sin embargo el 95\% en ambas regiones, manifiesta haber delineando la estrategia genérica competitiva y solo un 5\% menciona no haberlo establecido. La preparación de un plan estratégico no es una práctica generalizada entre los empresarios entrevistados.
La capacidad para movilizar recursos es esencial para la puesta en marcha de una empresa exitosa. La experiencia laboral previa y las redes de contactos ayudan a abrir las puertas a los recursos necesarios para iniciar las operaciones de la empresa. Más del 35\% de los empresarios de ambas localidades señalaron que la experiencia laboral previa los ayudó a obtener tecnología, relaciones y otros recur- 


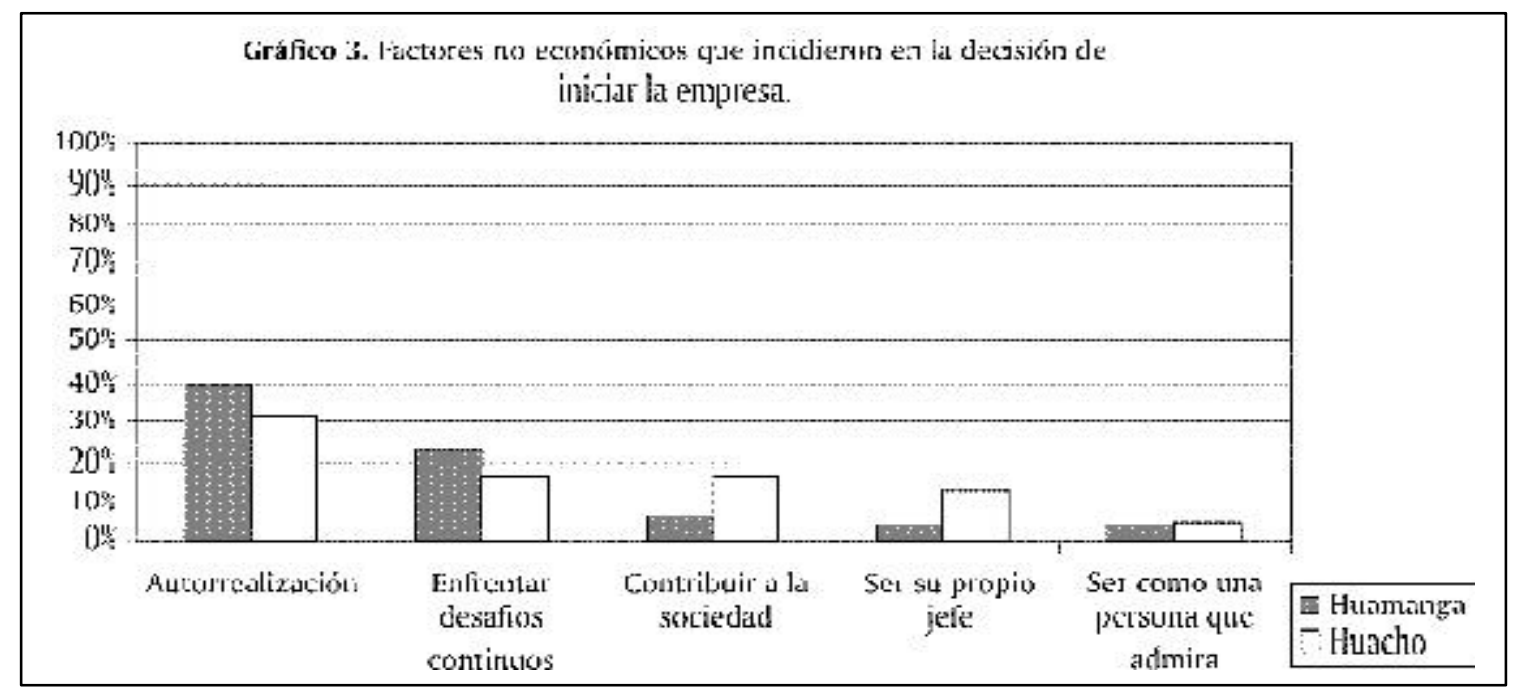

sos no financieros, incluyendo información, insumos y recursos materiales de operación para la producción.

$\mathrm{El}$ acceso a los recursos financieros es «un reto» para la puesta en marcha y el desarrollo inicial de las empresas. En ambas regiones los ahorros personales del empresario son la principal fuente de recursos para el lanzamiento del negocio. El 56\% de los emprendedores huachanos utiliza sus propios ahorros para lanzar su empresa, mientras que un 13\% usa recursos financieros provenientes de amigos y parientes; esto en ambas regiones. Las fuentes de financiamiento externo son los préstamos bancarios, que se encuentran más disponibles en Huacho que en Huamanga, donde la falta de financiamiento es uno de los mayores obstáculos para los emprendedores.

- La etapa de desarrollo inicial. En los tres primeros años de operación de la empresa, ambos sectores dicen enfrentar similares nive- les de competencia. La estrategia genérica empresarial predominante en Huacho está enfocada en la diferenciación y en Huamanga, en el liderazgo de bajo costo. En esta etapa el rango de fuentes de financiamiento que usan los emprendedores tiende a ampliarse. Las empresas nuevas ubicadas en Huacho recurren a créditos bancarios (comerciales) en mayor medida que en Huamanga.

La capacidad de resolver problemas en los primeros años es fundamental. Los empresarios huamanguinos enfrentan mayores dificultades para conseguir equipamiento adecuado y contratar trabajadores calificados, mientras que para los huachanos el problema principal radica en conseguir proveedores adecuados. Ambas regiones comparten el mismo tipo de problema en esta etapa: encontrar nuevos clientes y financiar y administrar el flujo de caja (Gráfico 4) .

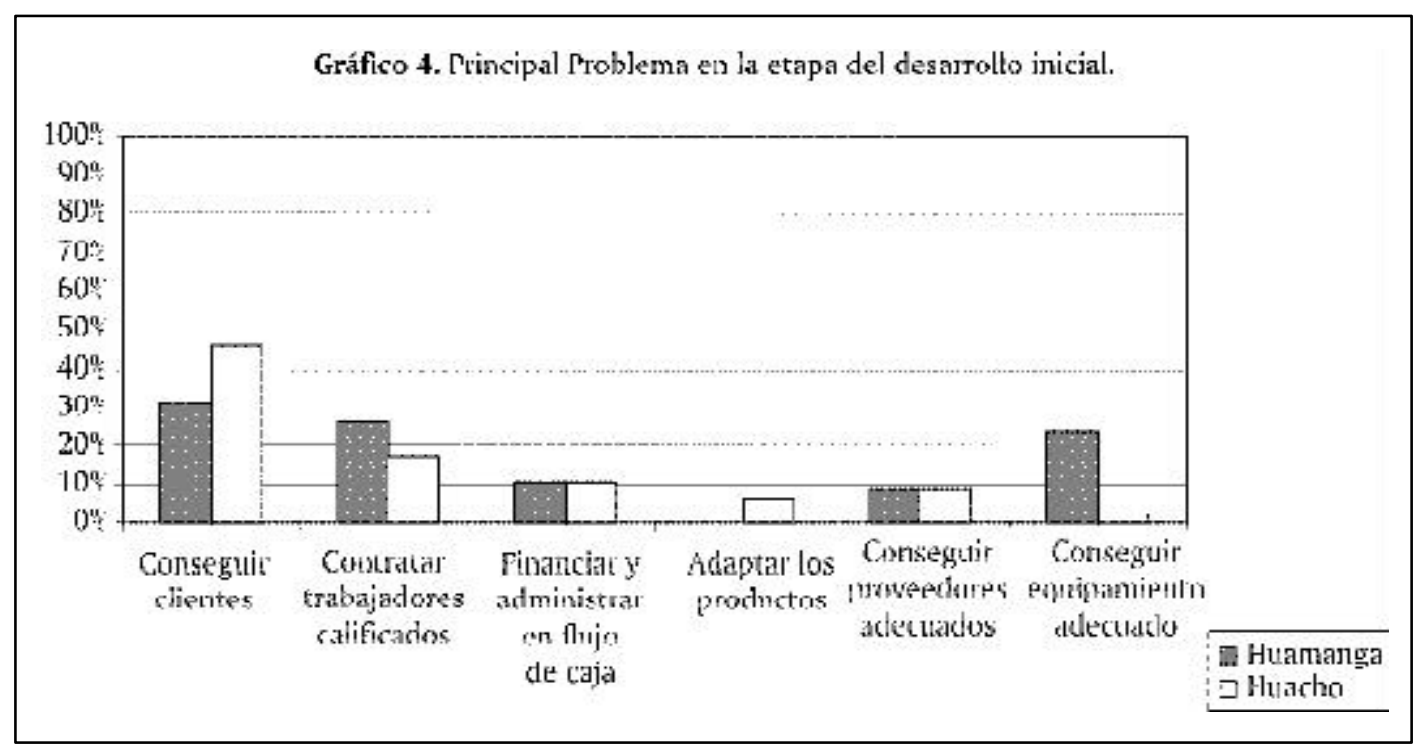


Más del 80\% de los empresarios de ambas regiones señalan que las instituciones formales existentes, como la Cámara de Comercio, asociaciones empresariales, universidades, centros de investigación y otros, no fueron capaces de brindar el asesoramiento necesario para resolver los problemas que enfrentaron durante el desarrollo inicial de sus negocios. Curiosamente la organización pública a través de PROMPYME fue capaz de asesorar a emprendedores de Huacho y Huamanga en razón del 9\% y $14 \%$ respectivamente durante el desarro110 inicial de sus negocios (Gráfico 5) .
Los factores que limitan la puesta en marcha involucran la escasez de financiamiento, con más incidencia en Huamanga; la complejidad burocrática para la formalización y los altos costos impositivos y regulatorios; esto último en ambas ciudades.

- Etapa de desarrollo inicial. Ambas localidades enfrentan similares niveles de competencia Los factores que motivan el desarrollo inicial de empresas están referidos a la experiencia previa, con mayor incidencia en los emprendedores de Huacho.

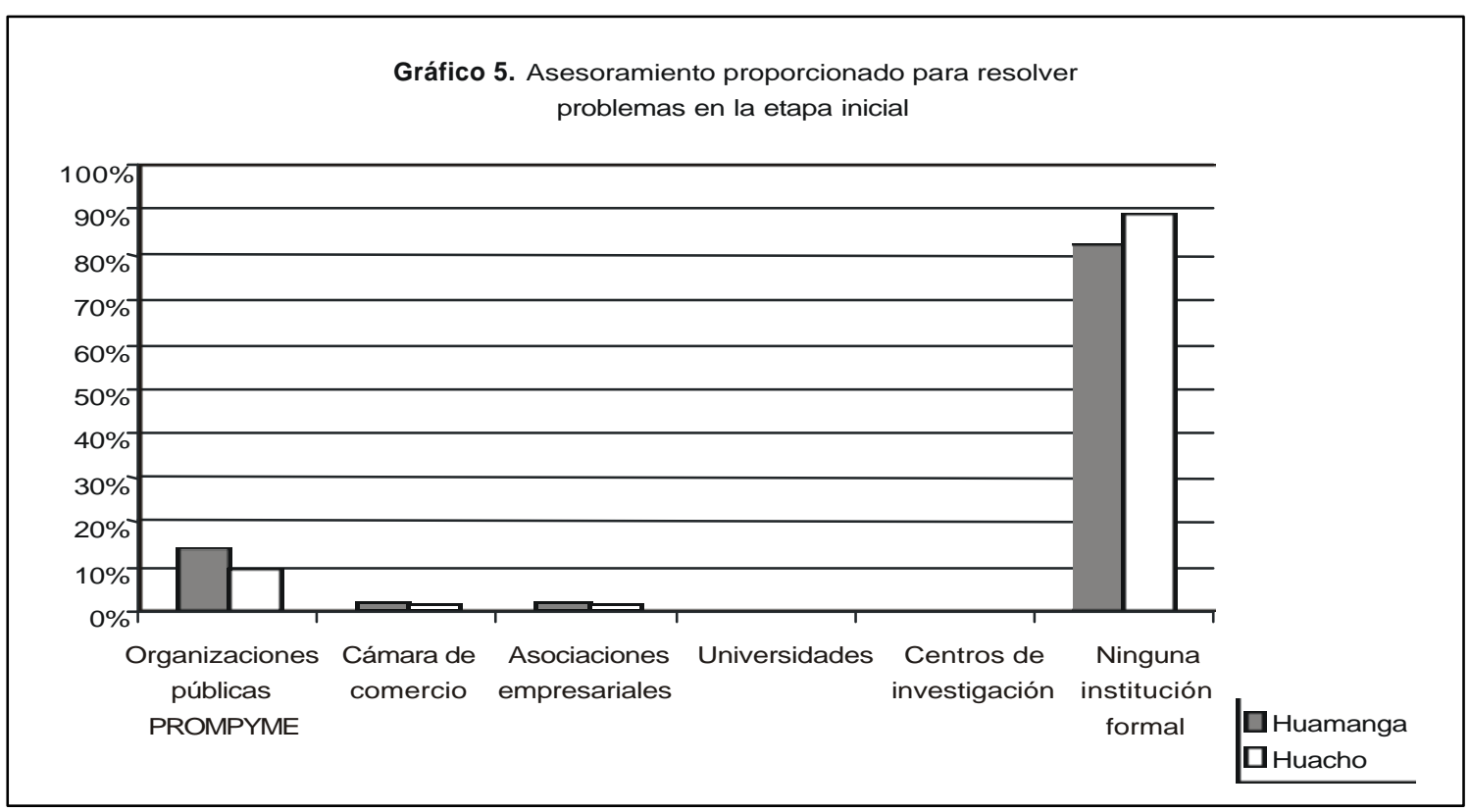

\section{ANÁLISIS Y DISCUSIÓN - CONCLUSIONES}

Las principales motivaciones que dan origen al ingreso de los emprendedores en el mundo de los negocios incluyen objetivos económicos y de desarrollo personal en tres etapas críticas:

- Etapa de gestación. En ambas localidades la mejora de ingresos es el principal motivo para crear un negocio y se cita con mayor frecuencia a la autorrealización como objetivo de tipo no económico y enfrentar desafíos continuos. El apoyo familiar es un aspecto positivo en la motivación del empresario, y la experiencia laboral previa ejerce un efecto motivacional decisivo.

- La etapa de puesta en marcha. Similares motivaciones que en la etapa de gestación. Aquí las motivaciones personales más importantes son la autorrealización personal, ser su propio jefe y mejorar el ingreso, características que prevalecen en ambas localidades.
Entre los motivos que limitan el avance empresarial, en los empresarios huamanguinos predominan dificultades para financiar flujos de caja e identificar proveedores adecuados, mientras que para los empresarios huachanos el principal problema radica en la contratación de la gerencia y en las relaciones organizacionales. Ambas regiones comparten el mismo tipo de problema: encontrar nuevos clientes y contratar trabajadores calificados con experiencia necesaria.

\section{NOTAS}

1 Argetelli, P. y Moori, Virginia. Desarrolloemprendedor: América Latina y la experiencia internacional. México, 2002.

2 Mantis, Mishahito. Empresarialidad en economías emergentes: creación y desarrollo de nuevas empresas en América Latina y el Este de Asia. BID, 2002.

3 Op.Cit

Schumpeter, Joseph. El espíritu emprendedor en las organizaciones. México, 1965. 
5 MC Clelland, David. El programa intraemprendedor y la búsqueda del cambio de actitud para impulsar la innovación empresarial. MC Graw Hill, 1961, pp. 76-78.

6 Gilder, George. Empresarialidad: Contribución al crecimiento y al bienestar económico. Ed. MC Graw Hill, 1984 .

7 Helcombe. Formación empresarial como factor del desarrollo económico. Lima, ILE, 1988.

8 Schumpeter, Joseph. 1965. Op. Cit.

9 Bygrave. The Portable MBA in Entreperneurchip. Segunda Edición. EE.UU., Jhon Wiloy \& Sons, InC., 1999.

10 Timmons, J. A. y Spinell, S. New Venture Creation Entrepreneurship for the 21st Century. EE.UU., MC Graw Hill e Irwin.

11 Dehter, Mario. El Perfil del Programa de Entrepreneurship. EE.UU., Babson School, 2001, pp. 81-83.

\section{BIBLIOGRAFÍA}

Argetelli, P. y Moori, Virginia. Desarrollo emprendedor: América Latina y la experiencia internacional. México, 2002.

Artículo. «El espíritu emprendedor en las organizaciones». En: Instituto Tecnológico Autónomo de México Informa - Boletín Electrónico. N. 2, año 1, enero-marzo, 2004.

Barrenechea, Carlos. El Centro Sur Andino: desafíos en la tradición de la sobrevivencia al desarrollo humano competitivo. CARE. Ayacucho, Sinapsis S. A., 2000.
Bygrave. The Portable MBA in Entrepreneurship. Segunda Edición. EE.UU., Jhon Wiloy \& Sons Inc., 1999.

Dehter, Mario. El Perfil del Programa de Entrepreneurship. EE.UU., Babson School, 2001.

Gilder, George. Empresarialidad: Contribución al crecimiento y al bienestar económico. Ed. Mc Graw-Hill, 1984.

Gilder, George. Nuevos emprendimientos y emprendedores ¿de qué depende su creación o supervivencia? Ed. McGraw-Hill, 1996.

Helcombe. Formación empresarial como factor del desarrollo económico. Lima, ILE, 1988.

Mantis, Mishahito. Empresarialidad en economías emergentes: creación y desarrollo de nuevas empresas en América Latina y el Este de Asia. BID, 2002 .

Mc Clelland, David. El programa intraemprendedor y la búsqueda del cambio de actitud para impulsar la innovación empresarial. McGrawHill, 1961.

Schumpeter, Joseph. El espíritu emprendedor en las organizaciones. México, 1965.

Timmons, J. A. y Spinell, S. New Venture Creation - Entrepreneurship for the $21^{\text {st }}$ Century. EE.UU., McGraw-Hill e Irwin. 